\title{
Efectividad del control simultaneo en las entidades de sector Educación de Lima Provincias-2020
}

Effectiveness of the simultaneous control services, in the entities of the Education Sector of Lima Provincies- 2020

\section{Mtra. Collantes Jiménez Haydee Emilia}

Mtra. En Gestión Pública y Gobernabilidad; cargo actual: Jefa de OCI organización donde labora: UGEL 12; correo electrónico: hcollantes@contraloria.gob.pe; urpiemilia@gmail.com; haydeecj11@hotmail.com; ORCID (0000-0002-6096-7083).

\section{Revista Iberoamericana de la Educación \\ Vol - Especial 12021 \\ e-ISSN: 2737-632x}

Resumen: El presente artículo trata acerca de la efectividad del control simultáneo, cuyo objetivo general fue determinar el nivel de Efectividad del control simultáneo en las Entidades de sector educación de Lima Provincias, se realizó el estudio debido a la gran importancia que hoy en día tiene las medidas de control en los entes públicas para prevenir y cautelar los bienes del estado. El presente artículo tiene un enfoque cuantitativo, tipo de investigación básica, diseño no experimental, descriptivo y transversal, se trabajó con una población de 70 empleados en entes públicas y se realizó la encuesta a través del cuestionario, el cual se validó con el Alpha de Cronbach con un valor de 0,971. Conclusiones de acuerdo a los resultados generales encontrados de la variable control simultáneo según los encuestados, tenemos que el $88.3 \%$ lo ve que este control es muy efectivo y el $11.7 \%$ lo ve el control efectivo. Los resultados de los objetivos específicos tenemos que el control concurrente que el $88.3 \%$ lo ve muy efectivo, el $11.7 \%$ lo ve efectivo, la visita de control lo ve que el $86.7 \%$ muy efectivo en tanto el $13.3 \%$ lo ve efectivo y orientación de oficio del control simultáneo el $85.0 \%$ considera que este control es muy efectivo y el $15.0 \%$ lo ve como efectivo.

Palabras clave 


\section{Órgano de Control Institucional}

Abstract: This article deals with the effectiveness of the simultaneous control, whose general objective was to determine the level of effectiveness of the simultaneous control in the Entities of the education sector of Lima Provinces, the study was carried out due to the great importance that measures have nowadays of control in public entities to prevent and protect state assets. This article has a quantitative approach, type of basic research, nonexperimental, descriptive and cross-sectional design, we worked with a population of 70 employees in public entities and the survey was carried out through the questionnaire, which was validated with the Alpha of Cronbach with a value of 0.971 . Conclusions according to the general results found of the simultaneous control variable according to the respondents, we have that $88.3 \%$ see that this control is very effective and $11.7 \%$ see it as effective control. The results of the specific objectives we have that the concurrent control that $88.3 \%$ see it very effective, $11.7 \%$ see it effective, the control visit sees it that $86.7 \%$ very effective while $13.3 \%$ see it effective and orientation of office of simultaneous control, $85.0 \%$ consider that this control is very effective and $15.0 \%$ see it as effective.

Introducción: El actual Articulo de Revisión titulada efectividad de servicios de control simultáneo, en las entidades del sector educación de Lima Provincias-2020, procura ser una contribución para fortalecer el control gubernamental en entes estatales del sector educación, adquirió como problema general ¿Cuál es el horizonte de Efectividad de servicios de control simultaneo en las entidades publicas de sector educacion de Lima Provincias2020?, el objetivo general, Establecer el horizonte de seguridad de servicios de control simultáneo, en las entes estatales del sector educación de lima provincias- 2020, objetivos específicos: Establecer el horizonte de 
efectividad del control concurrente del control simultáneo en entes estatales del sector educación de Lima Provincias - 2020, Comprobar el horizonte de la seguridad de la disposición de oficio del control simultáneo en entes estatales del sector educación de Lima Provincias - 2020, Comprobar el horizonte de la seguridad de la visita de inspección del control simultáneo de entes estatales del sector educación de Lima Provincias - 2020, el Hipótesis general: El trabajo de control en equipo en qué grado influyen el nivel de efectividad de servicios de control simultáneo en entes estatales del sector educación de Lima Provincias - 2020 y Hipótesis específicos: El servicio del control concurrente del control simultáneo influye el nivel de efectividad en entes estatales del sector cultura de Lima Provincias - 2020, El servicio del control visita del control simultáneo influye el nivel de efectividad en entes estatales del sector cultura - 2020, El servicio de orientación de oficio del control simultáneo influye el nivel de efectividad en entes estatales del sector educación de Lima Provincias - 2020.

En la coyuntura uno de los aspectos crecidamente significativos en el transcurso del trabajo estatal es investigar los medios y componentes obligatorios hacia la certificación, la transparencia y la intervención en todo lo que va relacionarse los bienes y prestación. Toda vez, Sin embargo, por las demostraciones y los efectos del servicio advertimos que es un argumento muy dificultoso de fiscalizar y hallamos variados atmosferas de corrupción y de egresos descomedidos, sobrevaluados en las disposiciones del sector estatal de Lima Provincias.

Por otro lado en los entes estatales, el inspección interno es un mecanismo que enlaza sus componentes, cuyo objetivo es salvaguardar los recursos públicos, asegurar la autenticidad de la información financiera y presupuestal a través de la rendición de cuentas, así como fomentar la eficiencia de las operaciones, siendo considerado una herramienta administrativa para cubrir 
sus objetivos con eficiencia, mejorar su desempeño y vigilar la aplicación de la normatividad en términos de trasparencia y rendición de cuentas, que no solo es aplicable a las finanzas sino a todas las áreas de una organización. Asimismo, es un ente adentro de cualquier estructura, que promueve el acatamiento de objetivos, normativa aplicable como leyes o reglamentos y reducción de gastos o pérdidas; sin embargo en el ejercicio prototipo ciertos inconvenientes reflejan, donde localizan servidores renuente para permitir premisas que administran a optimizar el ejercicio administrativo en sus instituciones donde laboran, a esto comprometemos encontrar un procedimiento idóneo, debemos extender un método de sensibilización, orientación y diligencia sobre el conocimiento del Sistema de vigilancia intrínseco y su importancia, conjuntamente día a día se exhibe en las instituciones públicas que toman decisiones en los procedimientos de control para la mejora en la gestión, además el diagnóstico a realizarse debe tener una visión del estado situación actual en consecuencia al procedimiento con el fin de una adecuada decisión al rendimiento profesional. Asimismo, los métodos de control son importante de prevenir y mejorar los procedimientos administrativos, teniendo en cuenta el método previo y simultaneo (realizados por los servidores del Sistema Nacional de Control-SNC, Órganos de Control Institucional-OCI), ante estos hechos se previenen la malversación de fondos públicos. Urge de gran cambio en lo ético profesional hacia los servidores y además sensibilizar con capacitaciones o talleres sobre temas de control para lograr los objetivos establecidos y no a modo de ampliar las funciones que desempeñan.

El actual artículo se justifica por el valor obtenido del estudio de inspección sincrónico, ante hechos de corrupción a nivel nacional, el cual se sustenta en el Reglas Habituales de Inspección Estatal de Normas Generales de Control Gubernamental-NGCG, aprobado por la Resolución de Contraloría N. ${ }^{\circ} 273$ - 
CG; y de acuerdo con el Art. $6^{\circ}$ al Art. $11^{\circ}$ y el $2^{\circ}$ párrafo del Ley N. ${ }^{\circ} 27785$, Ley Orgánica del Sistema Nacional de Control y de la Contraloría General de la República y la Directiva N. ${ }^{\circ}$ 002-CG/NORM, aprobado por la Resolución de Contraloría N. ${ }^{\circ} 115-2019-C G$, de 28 de marzo de 2019 y su modificatoria mediante Resolución de Contraloría N 144-2020-CG de 26 de mayo de 2020, y contribuciones de otros autores se puede sufragar a un excelente discernimiento de la normativa concerniente con la finalidad de coadyuvar a un excelente inspección de la misión estatal y certificar la nitidez y el completo trascurso en el servicio de los patrimonios y productos del sector estatal de Lima Provincias, en el sentido que a través de la motivación como eje principal se propone impulsar el fortalecimiento de servicios de inspección control simultaneo del control gubernamental. (Contraloría, 2002).

Se ha encaminado la dificultad como pedestal de nuestro Articulo de revisión desde las organizaciones públicas de sector educación a nivel de Lima provincias, tales como Unidades de Gestión Educativa Local-UGLs, entidades estatales, de manera especial con su equipo de Órganos de Control Institucional, Gerencia de Inspección Gubernamental de la Contraloría General de la Republica, quienes son los responsables de ejecutar el control gubernamental en todos sus actos, conforme a su plan de anual de controlPAC y sus responsabilidades inherentes de control y su mejora a partir de la normativa, textos y escritores que han coadyuvado el texto.

Los servicios de control, ejecutados por los Órganos del Sistema Nacional de Control-OSNC, son mediante diferentes tipos de control estatal, si bien estos son importantes, el investigador considera que, el nuevo enfoque del control gubernamental, mediante trabajos de control simultaneo, expanden a orientar su accionar de manera preventiva, que custodie la gestión 
estatal, con la finalidad de evitar situaciones que presenten riesgos que impidan, paralicen el desempeño de las metas y objetivos institucionales.

Por consiguiente anverso al contexto y por las ideas expuestas en los párrafos precedentes el propósito es averiguar la atribución al control simultaneo dentro de la ocupación profesional de los servidores en las entidades públicas de sector de educación, le valdrá como un instrumento de calidad a disposición del control gubernamental como metodologías hacia gestiones, enfocada en advertir conflictos de los métodos en las instituciones del sector público con vistas a emprender la validez, vigor, nitidez y patrimonio en las gestiones de las entes públicas de sector educación de Lima Provincias -2020, así prevenir y salvaguardar los recursos públicos e informar alteraciones en hechos de corrupción.

Para desenrollar la pesquisa se ha estimado el formalidad de la revista que considera lo siguiente: 1) Introducción donde se exponen las relativas justificaciones, el problema de averiguación a partir del contexto problemático, la enunciación del dificultad general de los delimitados, igualmente se ejecutó los objetivos generales y determinados y hipótesis, 2) Materiales y métodos, se esboza la metodología del artículo, se despliega la definición conceptual y estratégico de las variables en artículo; la sistemática, el tipo de estudio y diseño, retrato de la población, muestra y muestreo y la forma del procesamiento de los reseñas derivados, que se utilizó para el desarrollo de la exploración, el capítulo adonde se ostentan los antecedentes internacional y propios, la asientos teóricas, las desemejantes axiomas avecina de las variables así como la axioma de los indicadores, que desenvuelve el tipo de diseño de pesquisa, la ilustración conceptual y estratégica de las variables en disertación y paralización, el tipo de estudio y boceto, delineación de la urbe, modelo y muestreo, técnicas e instrumento de cosecha de testimonios, perfil del procesamiento de los datos alcanzados, que 
se manejó para el perfeccionamiento de la exploración, técnicas de exámenes de noticias, aspectos moralistas, 3) Resultados, existe consignado al progreso de los resultados 4) Discusión, en donde se plantean exponer la discusión, 5) Conclusiones, se tratan las conclusiones del articulo y Referencias.

\section{Materiales y métodos}

La investigación que presento es nivel básico, tipo descriptivo. Tamayo (2003) asevera que este tipo de investigación describe, registra, analiza e interpreta la naturaleza actual y la composición de los fenómenos. Tiene como objetivo principal presentar una interpretación correcta del fenómeno de estudio (p. 46). Desde la Perspectiva de Villegas (2010), la investigación pertenecería al tipo de investigación sustantiva descriptiva por que busca describir la realidad tal como es, tal como se presenta, en las condiciones y circunstancias en las que se presenta. (p. 87). El diseño de la investigación es no experimental dado que "la investigación que se realiza sin manipular deliberadamente variables. Es decir, se trata de estudios donde no hacemos variar en forma intencional las variables independientes para ver su efecto sobre otras variables" (Hernández et al., 2010, p. 149).

Tipo y diseño de investigación

Según Carrasco (2005), define que "Es la que no tiene propósitos aplicativos inmediatos, pues solo busca ampliar y profundizar el caudal de conocimientos científicos existentes acerca de la realidad.” (p. 43).

Zavala (2007), reveló que "el investigador debe diseñar un plan o estrategia concebido para responder a las preguntas de investigación, además para alcanzar sus objetivos de estudio y analizar la destreza de las hipótesis formuladas en un contexto en particular" (p. 97).

Igualmente, la pesquisa reincidirá en el diseño descriptivo simple, indaga y acopia investigación actual en relación de su tesis, la ocupación es narrar una 
particularidad, el examen estadístico será un análisis de frecuencias, gráficos semejantes. (IESPP CREA, 2010, p. 6)..

Investigación No Experimental

Hernández, (2014), definirse como la exploración que no maniobra intencionalmente las variables. Se trata de culturas donde no se transforman de forma proyectada las variables independientes para percibir su resultado de diferentes variables. Es prestar atención a circunstancias o fenómenos que resultan en su contexto nativo, subsiguientemente ilustrarse y/o examinar.

Investigación Transversal

Palella y Martins (2012), expresaron que se acopian datos en un solo momento, con la intención exponer las variables y aprender su acontecimiento e interrelación en ese momento.

Además, es de corte transversal ya que su propósito fue "describir variables y analizar su incidencia e interrelación en un momento dado. Es como tomar una fotografía de algo que sucede

Variables y Operacionalización

Definición operacional

Hernández, (2014) explica vinculación de medios, sistemáticas, ordenamientos, diligencias que van desenrollar para la comprobación de las variables y poder descifrar los datos emanados.

Definición operacional de la variable 1: Control simultaneo

Variable control simultaneo, precisa mediante tres dimensiones: Control Concurrente (14 items), Orientación de oficio (12 items), Visitas de control (8 items) y valoración y se calculó mediante una herramienta de escala ordinal tipo Likert.

Población, muestra y muestreo

Población 
La población, modelo y muestreo para Hernández, (2010) revela "la población o universo es un conjunto de todos los casos que concuerdan con determinadas especificaciones" (p.174)

La urbe en la investigación está compuesta por 70 trabajadores de diferentes entes de sector educación de Lima Provincias - 2020. El arquetipo de muestreo que empleamos en el modelo es no probabilístico, proporcionado que el procedimiento no es medio, si no que estriba del juicio de los arbitrajes del científico.

Técnicas e instrumentos de recolección de datos

Técnica

En relación conseguimos narrar como destreza al vinculado de diligencias, presupuestos para transportar un ejercicio concluyente.

Encuesta

Para el acopio de datos se utilizó la destreza de la encuesta.

Precisando Fontaines (2012):

Como una fusión entre la audiencia y examen estructurada, se determina por la elaboración de información de una audiencia encaminada a alcanzar pesquisa determinada delimitada en un etiqueta o herramienta de indagación, que puede ser desarrollada estadísticamente y igualmente, las derivaciones pueden ser extrapolados a la urbe. (p.145).

Instrumento

Hernández, 2014 indica "conjunto de preguntas respecto de una o más variables que se van a medir". (p. 216).

Instrumento 1: Variable de control simultáneo.

Nombre original: Cuestionario de control simultáneo.

Tipo de instrumento: cuestionario

Objetivo: Narrar especialidades de la variable control simultaneo de entes de sector enseñanza 
Dirección: Individual

Duración: 40 minutos

Significación: El cuestionario está concerniente a establecer la certeza de la inspección simultánea.

Estructura: El cuestionario consta de 36 ítems, con una escala de 05 alternativas de respuestas de opción múltiple, de tipo Likert, como: Nunca (1), Casi nunca (2), A veces (3), Casi siempre (4) y Siempre (5).

Procedimientos

Ratificación y confiablidad de la herramienta

Validez

Palella y Martins 2012, precisa "...el alejamiento de sesgos. Simboliza la correspondencia entre lo que se mide y aquello que verdaderamente se quiere medir" (p. 160)

\section{Confiabilidad}

Según Hernández, (2014), la confiabilidad de una instrumento de cálculo "es el valor en que un herramienta causa consecuencias consistentes y coherentes" (p. 200).

Se realizó la prueba piloto con 40 servidores de entes de sector enseñanza de la región Lima Provincias con la intención de evaluar la actuación de la herramienta al instante de recoger los testimonios y validar la consistencia del comprendido.

Es puntual mostrar que se esgrimió como prueba de confiabilidad el estadístico Alfa de Cronbach de tal modo, apreciar la firmeza interna del cuestionario los cuales se procesaron con el programa estadístico SPSS versión 23.0.

Método de análisis de datos

Se esgrimió de software la hoja de cálculo MS. Excel 2013 y el estadístico SPSS versión 23.0 para precisar y/o narrar la variable en exposición. 
Las consecuencias derivadas posteriormente del procesamiento estadístico las informaciones serán encarnadas en tablas e imágenes que suministren su comentario.

Las instrucciones precedentemente aludidas se elaborarán por el software MS. Excel 2013 y el estadístico SPSS versión 23.0.

Aspectos éticos

Actual exploración se encuentra respaldada en pesquisas, identificaciones y saberes definitivamente evidentes, derivados de obras, y carillas virtuales únicos con creencia significativas médulas académicos

\section{Resultados}

Los resultados generales encontrados de la variable control simultáneo según los encuestados, poseemos que el $88.3 \%$ reflexiona que este control es muy efectivo en proporción el $11.7 \%$ valora el control así que efectivo. En concordancia a las derivaciones de los objetivos específicos poseemos del control concurrente que el $88.3 \%$ reflexiona que este acto es muy efectivo, el $11.7 \%$ lo medita efectivo, respecto a la visita de control del se observa que el $86.7 \%$ considera que este tipo de control es muy efectivo en tanto el $13.3 \%$ lo ve como efectivo y en relación con la orientación de oficio del control simultáneo el $85.0 \%$ reflexiona que este control es muy efectivo y el $15.0 \%$ valora como efectivo. 


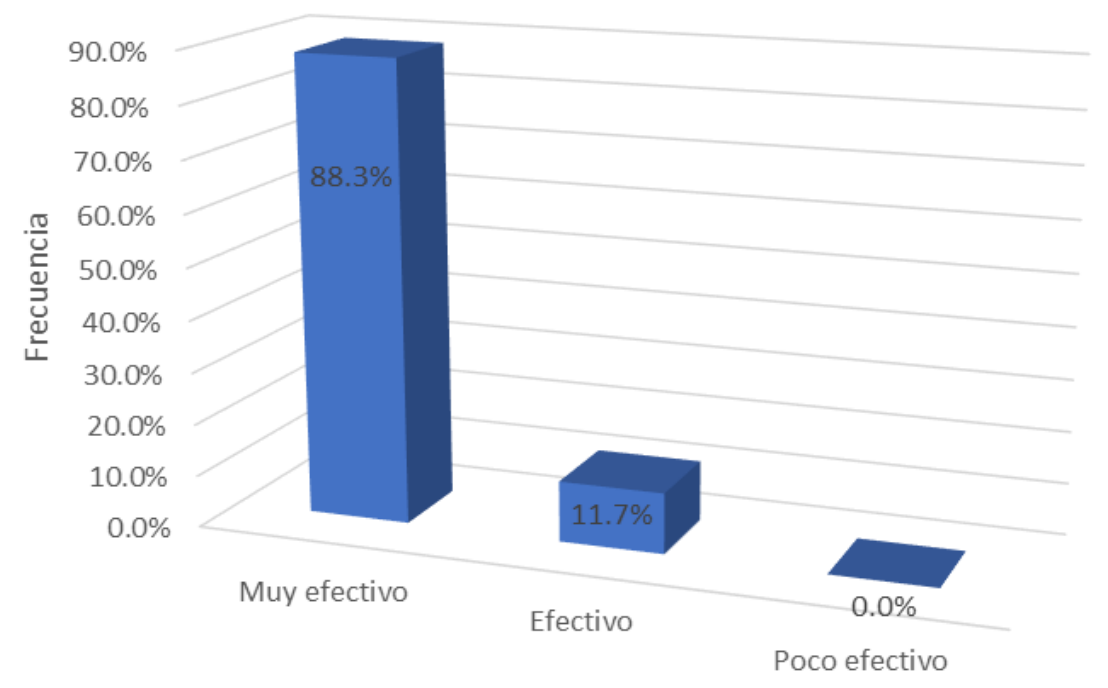

Figura 1. Nivel control simultáneo en la Control concurrente

De la figura 1 podemos apreciar los resultados respecto de la dimensión del control concurrente del control simultaneo que según los encuestados del control concurrente que el $88.3 \%$ reflexiona que este acto es muy efectivo, el $11.7 \%$ lo medita efectivo.

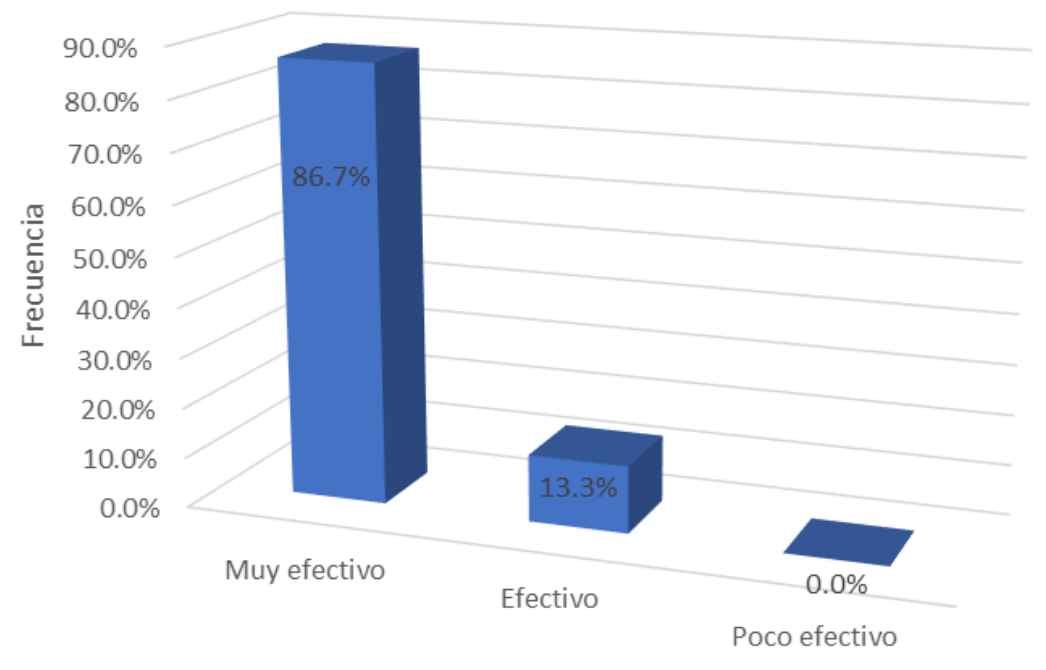

Figura 2. Nivel control simultáneo en la visita de control 
De la figura 2 podemos apreciar los resultados respecto de la dimensión de la visita de control del control simultaneo que según los encuestados observa que el $86.7 \%$ considera que este tipo de control es muy efectivo en tanto el $13.3 \%$ lo ve como efectivo

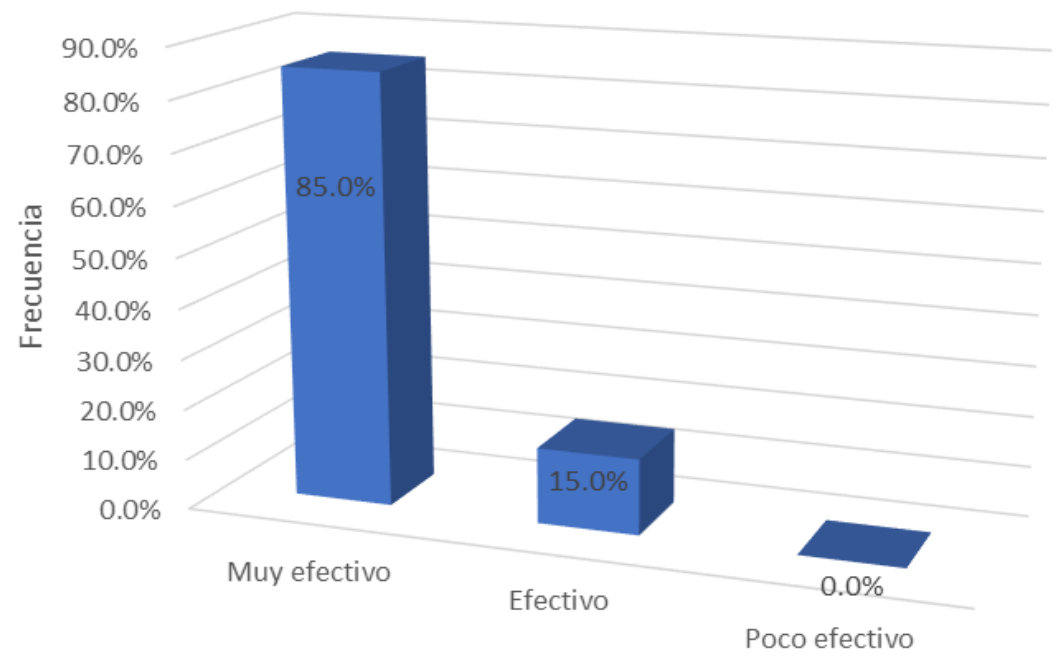

Figura 3. Nivel control simultaneo en la orientación de oficio

De la figura 3 podemos apreciar los resultados respecto de la dimensión orientación de oficio del control simultaneo que según los encuestados el $85.0 \%$ reflexiona que este acto es muy efectivo, el $15.0 \%$ lo ve como efectivo. 


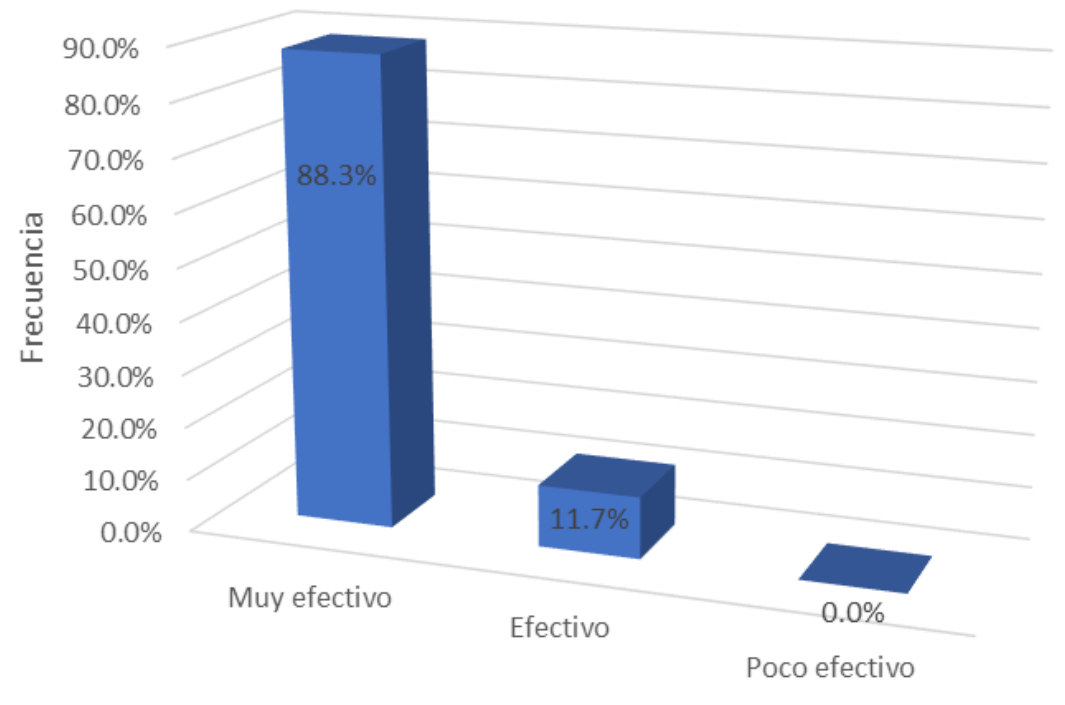

Figura 4. Nivel control simultáneo

De la figura 4 podemos apreciar los resultados respecto del variable control simultáneo que según los encuestados el $88.3 \%$ reflexiona que este control es muy efectivo en tanto el $11.7 \%$ estima el control como efectivo.

\section{Discusión}

De los resultados tenemos que de acuerdo al objetivo general que fue determinar el nivel de efectividad del control simultáneo en los entes de sector educación de Lima Provincias- 2020, tenemos que la variable control simultáneo según la apreciación de los encuestados el $88.3 \%$ considera que este tipo de control es muy efectivo en tanto el $11.7 \%$ lo aprecia como efectivo, en relación con los objetivos específicos que fueron comprobar el nivel de efectividad del control concurrente, la visita de control y la orientación de oficio en los entes de sector educación de Lima Provincias2020, tenemos que el control simultáneo según la apreciación de los encuestados el $88.3 \%$ reflexiona que este tipo de control es muy efectivo y el 11.7\% lo aprecia como efectivo, control concurrente según los encuestados medita que el $88.3 \%$ es muy efectivo, el $11.7 \%$ lo ve como efectivo, en 
correlación con la visita de control se observa que el $86.7 \%$ considera es muy efectivo en tanto el $13.3 \%$ lo ve como efectivo y la dimensión orientación según los encuestados el $85.0 \%$ reflexiona que este control es muy efectivo y el $15.0 \%$ recapacita como efectivo.

Arceda (2015), titula su tesis Efectividad de los procedimientos de control interno que se aplican en los espacios de gestión administrativa en la empresa agrícola "Jacinto López" S.A. del municipio de Jinotega durante el año 2015. El objetivo de la investigación es Evaluar la Efectividad de los Procedimientos de Control Interno que se emplean en los espacios de gestión administrativa en la Empresa Agrícola "Jacinto López S.A. del municipio de Jinotega durante el año 2015. El tipo de indagación es interpretativo. Los materiales utilizados fueron entrevista y pregunta de control interno. Se concluye que los recursos de la compañía son cedidos de forma oral a cada empleado con los que van a trabajar. La negociación de personal lo efectúa, según necesidad en cada espacio. Carencia de inspección de entrada y salida del personal. Los arqueos de caja se ejecutan casualmente. Los ingresos del día ausencia de depositados, los mismos son manejados egresos.

Cambier (2015), titula su tesis Evaluación del desempeño de la inspección interna de una oficina de registro de un ente de estudios superiores permanente en técnicas de auditoría interna. El objetivo de la indagación es valorar el nivel de ejercicio del control interno de la oficina de registro de una institución de estudios superiores. El tipo de indagación utilizado fue el evaluativo. La muestra se llevó a cabo con 34 personas de cinco universidades. Como herramienta se manejaron la encuesta y la entrevista. De acuerdo a la averiguación de percepción referente a la dimensión sobre el monitoreo de actividades y las entrevistas informales a los auditores entrevistados se puede concluir que solo en dos representan el $40 \%$ de un total de 5 de las universidades de las evaluadas, procesan reportes de auditoría 
los cuales son manifiestos a las autoridades universitarias, las cuales tramitan los mismos a los evaluados para la corrección de desviación o mejoras de procesos. El objetivo y alcance de la misión de la IES se cumple y a los grupos de interés, lo cual representa la base para crear estrategias de mejoramiento de sus políticas la enseñanza, la investigación y la gestión. Por otra parte El IIA Global confirma el informe de auditoría proporcionar garantía a la alta Dirección y al Consejo con respecto a los métodos de gobierno, la gestión de riesgos y la inspección.

Salazar (2014), titula su tesis El control interno: instrumento indefectible para el fortalecimiento de las capacidades de la dirección estatal de hoy. El objetivo de la exploración es estar al tanto cómo se ejecuta el control interno en las desemejantes instituciones del Ente. El prototipo de indagación es descriptivo. Se concluye que el actual discernimiento de Control Interno se encuadra dentro de una perspectiva de control completo, donde la inspección del mandato estatal y la demostración del ejercicio de las normas legales, se realiza a nivel interno y externo del ente. El Control Interno, pesquisa que la Alta Dirección, los gestores y servidores de cada ente, efectúen ejercicios de reserva previa, simultánea y posterior a sus acciones, con el propósito de la querencia de sus recursos, bienes y operaciones, este encaminado a la obtención de los objetivos, metas y misión de cada entidad. La Contraloría General, como órgano técnico competente de control gubernamental, ha determinado la base de una gestión de riesgos las entidades deben implementar los controles internos que sean necesarios para la consecución de los objetivos, metas y misión corporativo, lo cual además beneficia el desempeño de objetivos gerenciales relacionados con el buen manejo de los recursos públicos, destacándose entre ellos la nitidez, la organización interna, la rendición de cuentas y el control posterior. 


\section{Conclusiones}

1. De acuerdo con los resultados podemos apreciar que la variable control simultáneo según la valoración de los encuestados el $88.3 \%$ reflexiona que este arquetipo de control es muy efectivo en proporción el $11.7 \%$ lo valora como efectivo; de las derivaciones relación de la dimensión del control concurrente del control simultáneo según los encuestados se posee que el $88.3 \%$ reflexiona que este hecho es muy efectivo, el $11.7 \%$ piensa como efectivo; resultados relación de la dimensión de la visita de control de la inspección simultánea según los encuestados se ve que el $86.7 \%$ reflexiona que este tipo de inspección es muy efectivo y el 13.3\% estima como efectivo; resultados respecto de la dimensión orientación de oficio del control simultáneo según los encuestados el $85.0 \%$ razona que esta inspección es muy efectiva y el $15.0 \%$ discurre como efectivo.

2. Recomendar a los jefes de Órgano de Control Institucional de los entes del Sistema Nacional de Control, afín de que promuevan ejecución del ejercicio del Control Simultaneo en todas sus modalidades, para prevenir la corrupción.

\section{Referencias}

Gardner, H. (2001). La inteligencia reformulada. Las inteligencias múltiples en el siglo XXI. Buenos Aires, Argentina: Paidós.

Hernández, R., Fernández, C. \& Baptista, P. (2010). Metodología de la investigación. Ciudad de México, México: McGraw-Hill.

Huidobro, T. (2002). Una definición de la creatividad a través del estudio de 24 autores seleccionados. Madrid, España: Universidad Complutense de Madrid.

Lobo, N. \& Santos, C. (1990). Psicología del aprendizaje. Bogotá, Colombia: Universidad Santo Tomás. 
Saiz, C. \& Fernández, S. (2012). Pensamiento crítico y aprendizaje basado en problemas cotidianos. Revista de Docencia Universitaria, 10(3), 2-17.

Sastre-Riba, S. (2011). Funcionamiento metacognitivo en niños con altas capacidades. Revista de Neurología, S2(Supl. 1), 11-18.

Sternberg, R.J. \& Lubart, T.I. (1999). Manual de creatividad 1. Cambridge, Inglaterra: Universidad de Cambridge.

Torre, S. (1997). Creatividad y formación: identificación, diseño y evaluación. Ciudad de México, México: Trillas.

Torrance, E.P. (1972). Can We Teach Children to Think Creatively? The Journal of Creative Behavior, 6(2), 114-143.

Alarcón. (2013). Gestión educativa y calidad de la educación en instituciones privadas en Lima Metropolitana.

http://www.repositorioacademico.usmp.edu.pe/bitstream/usmp/1168/1/alarc on_msz.pdf

Arboleda, (2011). El Modelo estándar de control interno (MECI) como herramienta que facilita el proceso de gestión escolar en una Institución Educativa. Colombia: Repositorio UNAL.

https://repositorio.unal.edu.co/bitstream/handle/unal/7205/gustavowilliamar boledaortiz.2011.pdf?sequence $=1 \&$ isAllowed $=\mathrm{y}$ Arceda, (2015) Efectividad de los Procedimientos de Control Interno que se aplican en las áreas de administración y contabilidad en la Empresa Agrícola “Jacinto López” S.A. Nicaragua: Repositorio Universidad Nacional Autonoma de Nicaragua.

https://core.ac.uk/download/pdf/53104039.pdf

Ballena, (2015). Implementación del Órgano de Control Institucional como herramienta de gestión en la Municipalidad distrital de Chao-La Libertad año 2014. Dspace UNITRU. Trujillo, Perú: Google Chrome. 
http://dspace.unitru.edu.pe/bitstream/handle/UNITRU/2437/TESIS\%20MA ESTRIA\%20$\% 20 \mathrm{~V} \% \mathrm{c} 3 \%$ a1 squez $\% 20$ Ballena $\% 2 \mathrm{c} \% 20$ Cynthya.pdf?sequence=1\&isAllo wed $=\mathrm{y}$

Camus, (2017). Influencia del Control Interno en la Gestión Administrativa de la Dirección Sub Regional de Salud Huallaga Central, 2016. Repositorio UCV. Perú: Google Chrome. Obtenido de https://repositorio.ucv.edu.pe/bitstream/handle/20.500.12692/1578/valera_c n.pdf? sequence $=1 \&$ isAllowed $=\mathrm{y}$

Castellón, (2015). Efectividad de los procedimientos de control interno que se aplican en las áreas de administración y contabilidad en la empresa agrícola 'Jacinto López' S.A. del municipio de Jinotega durante el año 2014. Repositorio UNAN. Matagalpa, Nicaragua: Google Chrome. Obtenido de https://repositorio.unan.edu.ni/1762/1/5320.pdf

Catalán, L. K. (2014). El control interno: herramienta indispensable para el fortalecimiento de las capacidades de la gerencia pública de hoy. Perú. Obtenido de http://hdl.handle.net/20.500.12404/5543

Cevasco, S. E. (2017). Desempeño laboral del personal administrativo nombrado de la Municipalidad Provincial del Santa - Chimbote 2017. Repositorio UCV. Perú: Google Chrome. Obtenido de https://repositorio.ucv.edu.pe/bitstream/handle/20.500.12692/12001/medina _cs.pdf?sequence $=1 \&$ isAllowed $=\mathrm{y}$

Directiva N¹3-2016-CG/GPROD, párr. 6.2. (13 de Mayo de 2016). Lima, Perú. Obtenido de https://busquedas.elperuano.pe/normaslegales/apruebanla-directiva-n-013-2016-cggprod-denominada-impl-resolucion-no-1492016-cg-y-directiva-n-013-2016-cggprod-1380288-1/

Gil, H. (2015). Sistema de estructuración integral de control interno para el área de inventario del sector fabricante de carrocerías ubicado en el Estado 
Aragua. Semantic Scholar. Google Chrome. Obtenido de https://pdfs.semanticscholar.org/384b/506cae0341cb435789f1d3eeaddcd29 80e05.pdf?_ga=2.131172559.15694123.16108228981423520246.1610822898

Guamán, S. F. (2016). Implementación de un Sistema de Control Interno para la estación de servicios la Argelia de la ciudad Loja. Dspace UNL. Loja, Ecuador: Google Chrome. Obtenido de https://dspace.unl.edu.ec/jspui/bitstream/123456789/10101/1/Tesis\%20Lista \%20Silvana.pdf

Guba, E., \& Lincoln, Y. (s.f.). "Paradigmas en competencia en la investigación cualitativa.". Obtenido de http://sgpwe.izt.uam.mx/pages/egt/Cursos/MetodoLicIII/7_Guba_Lincoln_P aradigmas.pdf

Collantes, H. E. (2017). "Efectividad del control simultaneo en la Unidad de Gestión Educativa Local $\mathrm{N}^{\circ} 12$. Canta - 2016. Perú. Obtenido de https://repositorio.ucv.edu.pe/bitstream/handle/20.500.12692/23542/Collant es_JHE.pdf?sequence $=4 \&$ isAllowed $=\mathrm{y}$

Ley 27785. (22 de Julio de 2002). Lima, Perú. Obtenido de https://cdn.www.gob.pe/uploads/document/file/431141/Ley-27785.pdf

Marmolejos, E. E. (2015). Evaluación del cumplimiento del control interno de un departamento de registro de una entidad de estudios superiores basado en técnicas de auditoría interna. España: Repositorio de la Universidad de Sevilla. Obtenido de https://idus.us.es/bitstream/handle/11441/32856/Tesis\%20Enrique\%20\%20 Cambier\%20evaluaci\%c3\%b3n\%20Cumplimiento\%20Control\%20Interno $\% 20 \mathrm{dpto} \% 20$ Registro.pdf?sequence $=1 \&$ isAllowed $=\mathrm{y}$

Ortiz, G. W. (2011). El Modelo estándar de control interno (MECI) como herramienta que facilita el proceso de gestión escolar en una Institución 
Educativa. Repositorio UNAL. Medellin, Colombia: Google Chrome. Obtenido

de

https://repositorio.unal.edu.co/bitstream/handle/unal/7205/gustavowilliamar boledaortiz.2011.pdf?sequence $=1 \&$ isAllowed $=\mathrm{y}$

Poveda, J. G., Puente Tituaña, S. P., \& Vera, P. Y. (2016). Importancia del Control Interno en la Gestión Pública. Dialnet. Guayaquil, Ecuador: Google Chrome. Obtenido

de https://dialnet.unirioja.es/descarga/articulo/5833405.pdf

Resolución Contraloría N³20-2006-CG, 2006, art. 1. (30 de Octubre de 2006). Lima, Perú. Obtenido de http://doc.contraloria.gob.pe/libros/2/pdf/RC_320_2006_CG.pdf Resolución de Contraloría N. o 115-2019-CG. (28 de Marzo de 2019). Lima, Perú. Obtenido de https://busquedas.elperuano.pe/download/url/modificanlas-normas-generales-de-control-gubernamental-y-ap-resolucion-no-1152019-cg-1755413-1

Ricoy Lorenzo, C. (2006). "Contribución sobre los paradigmas de investigación.". Santa María, Brasil. Obtenido de https://www.redalyc.org/pdf/1171/117117257002.pdf

Salazar Catalán, L. K. (27 de Agosto de 2014). "El control interno: siendo instrumento indispensable para el fortalecimiento de las capacidades de la gerencia pública de hoy.”. Lima, Perú. Obtenido de http://tesis.pucp.edu.pe/repositorio/bitstream/handle/20.500.12404/5543/SA LAZAR_CATALAN_LESLY_KARIN_CONTROL.pdf?sequence=1\&isAll owed $=\mathrm{y}$

Sánchez, A. E. (2019). Control Interno y Gestión Administrativa en la Municipalidad Distrital de Ocongate, 2017. Repositorio UNSAAC. Cusco, Perú: Google Chrome. Obtenido de 
http://repositorio.unsaac.edu.pe/bitstream/handle/UNSAAC/4558/253T2019 1121_TC.pdf? sequence $=1 \&$ isAllowed $=\mathrm{y}$

Villacorta, J. R. (2016). Sistema de Control Interno y su relación con el desempeño laboral de los colaboradores de la oficina de Gestión de las Personas del Gobierno Regional de San Martín, 2016. Repositorio UCV. Tarapoto, Perú: Google Chrome. Obtenido de https://repositorio.ucv.edu.pe/bitstream/handle/20.500.12692/852/rivera_vj. pdf? sequence $=1 \&$ isAllowed $=\mathrm{y}$

Zafra Gutiérrez, J. A. (2010). Metodología para evaluar la efectividad del diseño y operación de los controles en la realización de auditorías basadas en riesgos. Colombia: Repositorio UNAL. Obtenido de https://repositorio.unal.edu.co/bitstream/handle/unal/7577/98559963.2011.p df? sequence $=1$ \&isAllowed $=y$

Alarcón, S. (2013). Gestión educativa y calidad de la educación en instituciones

privadas en Lima Metropolitana.

http://www.repositorioacademico.usmp.edu.pe/bitstream/usmp/1168/1/alarc on_msz.pdf

Arboleda, G. (2011). El Modelo estándar de control interno (MECI) como herramienta que facilita el proceso de gestión escolar en una Institución Educativa.

http://www.bdigital.unal.edu.co/3538/1/gustavowilliamarboledaortiz.2011.p df

Arceda, S. (2015). Efectividad de los procedimientos de control interno que se aplican en las áreas de administración y contabilidad en la empresa agrícola "Jacinto López" S.A. del municipio de Jinotega durante el año 2014. http://repositorio.unan.edu.ni/1762/1/5320.pdf 
Cambier, E. (2015). Evaluación del cumplimiento del control interno de un departamento de registro de una entidad de estudios superiores basado en técnicas de auditoría interna.

https://idus.us.es/xmlui/bitstream/handle/11441/32856/Tesis\%20Enrique\%2 0\%20Cambier\%20evaluaci\%C3\%B3n\%20Cumplimiento\%20Control\%20In terno $\% 20$ dpto $\% 20$ Registro.pdf?sequence $=1$

Díaz, J. (2010). Influencia del Programa de Control Interno "CAMINO AL EXITO”, basado en el Pensamiento Estratégico, en la gestión administrativa de la Institución Educativa PNP. Mariano Santos Mateos”.

http://erp.uladech.edu.pe/archivos/01/01018/portadas/18648.pdf

Munguía, J. (s/f). Implementación de los sistemas de control interno en la administración pública municipal.

http://ce.jalisco.gob.mx/sites/ce.jalisco.gob.mx/files/munguia_hernandez_i mplementacion_de_los_sistemas_de_ci.pdf

Puertas, D. (2016). El control interno y su influencia en la administración de las municipalidades del Perú: caso Municipalidad Distrital de Nuevo Chimbote.

http://repositorio.uladech.edu.pe/bitstream/handle/123456789/934/CONTR

OL_INTERNO_ADMINISTRACION_PUBLICA_PUERTAS_RODRIGU

EZ_DELIA_VANESSA.pdf?sequence $=4$

Salazar, L. (2014). El control interno: herramienta indispensable para el

fortalecimiento de las capacidades de la gerencia pública de hoy.

file://C:/Users/Downloads/SALAZAR_CATALAN_LESLY_KARIN_CO

NTROL.pdf

Sotomayor, J. (2009). El control gubernamental y el sistema de control interno en el Perú: análisis crítico.

http://www.repositorioacademico.usmp.edu.pe/bitstream/usmp/341/1/sotom ayor_cj.pdf 
Shack, J. (2015). Los problemas en la implementación de una estrategia: análisis de la implementación de la Ley $\mathrm{N}^{\circ} 29555$ que incorpora plazas y presupuesto de los órganos de control institucional a la Contraloría General de la República periodo 2011 - 2014.

file://C:/Users/Downloads/SHACK_MURO_JORGE_RICARDO_PROBL EMAS.pdf 